\title{
Practice Spotlight: Pharmacist in a Chronic Kidney Disease Clinic
}

\author{
Angela McKinnon, BSP ACPR \\ Clinical Pharmacist \\ Chronic Kidney Disease Clinic \\ St Paul's Hospital \\ Saskatoon, Saskatchewan
}

$\mathrm{T}$ The Chronic Kidney Disease Program is a multidisciplinary outpatient clinic and outreach program at St Paul's Hospital in Saskatoon, Saskatchewan. The clinic opened in July 2001 with a vision to care for, educate, and support individuals and their families along the continuum of chronic kidney disease to achieve optimal outcomes. The clinic serves the northern half of the province and as of September 2010 had about 550 patients. The program's health care team has grown substantially since 2001 and now consists of nephrologists, registered nurses, an Aboriginal health educator, a dialysis access nurse, a dietician, a diabetes clinician, a social worker, an exercise therapist, a pharmacist, and support staff. The clinical pharmacist position (1 full-time equivalent [FTE]) was created in mid-2008, at the request of the hospital's department of Renal Services, and Angela McKinnon has held the position since then. Renal Services has an additional 4.3 FTE pharmacist positions in other areas (Hemodialysis, Home Therapies, and Transplant).

The Chronic Kidney Disease Clinic has a diverse patient population, and the clinic team faces many challenges in providing patient care, including linguistic and cultural barriers and financial and travel issues. Most patients have stage 3 to 5 chronic kidney disease (creatinine clearance less than $60 \mathrm{~mL} / \mathrm{min}$ ) due to a variety of causes. The patients range in age from 18 to over 90 years, and more than half (52\%) have diabetes mellitus. The team works with patients to slow the progression of their disease and to manage the associated symptoms, educating them about treatment options as the disease progresses. The clinic pharmacist provides a number of services to both patients and other members of the team.

Each patient is referred to the Chronic Kidney Disease Clinic by his or her nephrologist. At the initial appointment, the patient meets with all team members for a needs assessment. The pharmacist obtains the patient's medication and allergy histories. The pharmacist also educates the patient about the importance of medication management in chronic kidney disease, adherence to drug regimens, and the potential risks of nephrotoxic medications. At subsequent visits, the patient is seen by specific team members, as appropriate to the person's laboratory results or as requested by the patient or other team members. The pharmacist continually assesses drug therapy for efficacy and adverse effects, using laboratory results, the results of physical examinations performed during clinic visits, and information obtained during phone conversations with the patient. The main areas of focus for the chronic kidney disease pharmacist are management of anemia, monitoring for hypertension, reduction of cardiovascular risk, adjustment of doses, and recommendations relating to medications that are eliminated renally. Drug coverage and supply issues involve communication with community pharmacists and the office of the provincial health plan. The chronic kidney disease pharmacist deals with drug information requests from team members and other health care providers. Management of inventory and reporting (to the public health department) of vaccinations performed in the clinic are also the pharmacist's responsibility. Other members of the team participate in the patient education classes, which are offered throughout the year to provide patients with self-management information.

Another important component of the Chronic Kidney Disease Program is the Community Outreach Program. Team members travel to communities in central and northern Saskatchewan to promote awareness of chronic kidney disease to both patients and health care providers. The team provides information about prevention to at-risk populations through community-based activities and partners with health care providers to increase awareness and understanding of risk factors and screening for chronic kidney disease. To date, the pharmacist has had little involvement in community outreach, but there are plans to include the pharmacist in the future.

The Renal Services department at St Paul's Hospital offers a unique opportunity for collaboration between pharmacists. Renal Services pharmacists confer regularly and work together to provide seamless care as patients move through the continuum of chronic kidney disease. Communication with inpatient pharmacists to facilitate medication reconciliation at the time of patients' admission and discharge is another 
important responsibility of the Renal Services pharmacists. The Renal Services practice site gives pharmacy students and residents an opportunity to see patients as they move through the health care system and to understand the pharmacist's role within several health care teams.

The role of the chronic kidney disease pharmacist will advance as the Saskatchewan College of Pharmacists expands the clinical role of pharmacists through prescriptive authority. Collaborative prescribing agreements will enhance patients' access to care and medication management. One area of focus for collaborative prescribing in the Chronic Kidney Disease Clinic is management of anemia. Patients will receive optimal management and delivery of care as team members continue to expand their roles.
The Practice Spotlight series highlights the accomplishments of Canadian pharmacists with unique practices in hospitals and related health care settings. If you have a unique or innovative practice, or you know someone else who should be profiled, please submit your contact information to Mary Ensom, Editor of CJHP (cjhpedit@cshp.ca), and one of our Associate Editors will be in touch with you.

\section{Fancy yourself a photographer?}

The Canadian Journal of Hospital Pharmacy is looking for photographs for the cover of the Journal.

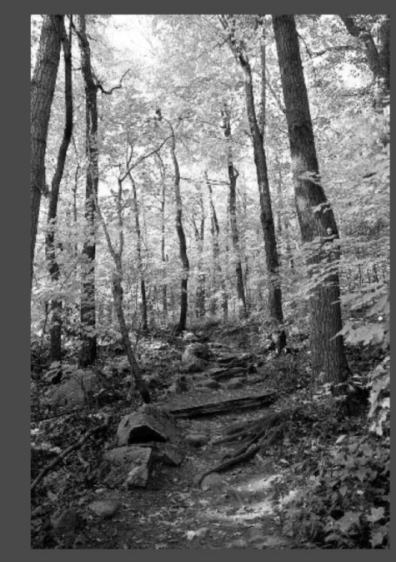
The photo theme for 2011 is:

\section{Canadian Landscapes and Scenery}

Interested participants are asked to submit a digital photo or group of photos for selection along with a short (max 150 words) write-up about the location of the photo, the date and time of the photo, and the type of equipment used. Be sure to include any stories or details that make this photograph or location unique!

Entries can be submitted to Colleen Drake, Publications Administrator, at cdrake@cshp.ca. 